\title{
Energy Efficient Spectrum Sensing Techniques for Cognitive Radio Networks: A Survey
}

\author{
Jayakrishna P. S. \\ $P G$ student \\ Department of ECE \\ NSS college of Engineering \\ Palakkad
}

\author{
Greshma V. \\ PG student \\ Department of ECE \\ NSS college of Engineering \\ Palakkad
}

\author{
T. Sudha \\ Professor \\ Department of ECE \\ NSS college of Engineering \\ Palakkad
}

\begin{abstract}
Cognitive radio has emerged as a tempting solution for the spectrum scarcity problem. This article focuses on the recent trends in energy efficient spectrum sensing techniques for the Cognitive Radio (CR) technology. The increasing demand of cognitive radio and its application increases the urge to make the emerging technologies as energy efficient as possible. Spectrum sensing which is one of the most complex and power intensive tasks in a cognitive radio system when made energy efficient increases the longevity of the network. This survey focuses on the new and efficient energy aware sensing techniques for cognitive radio networks and compares them.
\end{abstract}

\section{Keywords}

Cognitive radio, Spectrum sensing, Energy efficient sensing, Censoring, Sleeping, Sequential detection, Confidence voting, Cluster collect forwarding, Compressive sensing, RL based sensing, History assisted sensing, WSN assisted sensing, Trust based sensing.

\section{INTRODUCTION}

Cognitive radio [1] proves the technical solution for the issuance of spectrum scarcity for wireless communication. They can successfully deal with the growing demand and scarcity of the wireless spectrum. The resource constraints of the batteries, heating effect of the devices and the increased emission of the greenhouse gases increases the urge to minimize the energy consumption of the cognitive radios. There are several ways in which energy can be saved in cognitive radio networks. In this paper few of the new and emerging techniques for energy efficient spectrum sensing are discussed.

\section{ENERGY EFFICIENT SPECTRUM SENSING}

The SS process increases the energy consumption of sensing devices [2]. More often these devices will be energy constrained. Thus excessive SS, although critical for providingaccurate information about the radio environment, may lead to premature depletion of the sensing devices battery and consequently shorten its lifetime. Energy efficiency is thus a pertinent issue in a CRN. As the number of wireless devices and equipment continue to increase, there will always be a corresponding increase in the demand for more energy supply and a constant pressure in crafting out more energyefficient devices. Global warming has also become an important factor not to be overlooked in recent times and most government agencies, network service providers, manufacturers of network devices and also users are now disturbed about the energy efficiency issues of wireless devices than they used to. The importance in optimizing energy efficiency in cognitive radio networks are numerous but most of them points to the issue of design, green communications policy, savings as regards to monetary cost and end users gratification and fulfillment. The more the energy being expended a wireless device, the more the heat due to the fact that energy used up in wireless devices gives rise to heat. Environmental issues such as green-house gas problem have also been a major source of concern to various government agencies around the world. The more energy being used, the more green-house gas is being produced. Due to this reason, a lot of compulsory and non-compulsory standards now necessitate wireless devices to be more energy efficient. Some of the energy efficient spectrum sensing techniques are discussed below.

\subsection{Censoring}

In a cooperative spectrum sensing scenario, each cognitive radio consumes energy mainly on sensing the spectrum and then transmitting the raw or processed data to the FC. The idea behind distributed detection with censoring sensors lies in the fact that not all the local decision results are informative for the FC. Therefore, the transmission energy can be saved by avoiding sensors with not-informative results from communicating with the FC [3]. The censoring decision rule is employed to reduce the number of bits sent to the fusion center and so the bandwidth occupancy of the cognitive radio network. Each sensor calculates the energy of the collected samples and if it is deemed informative, then a bit indicating presence or absence of the primary user is sent to the FC. In case if not coming in the informative region, no decision is made and no bit is sent to the FC. This way, the number of transmissions is reduced and so is the transmission energy.

\subsection{Sleeping}

Sleeping or on/off sensing [4] is a power saving mechanism in which every cognitive radio randomly turns off its sensing device with a probability $\mathrm{m}$, the sleeping rate. The advantage of sleeping over censoring is that the cognitive radios that are asleep do not waste any sensing or transmission power, whereas in censoring all the cognitive radios have to spend energy on sensing. Sleeping has generally been applied in combination with censoring.

\subsection{Sequential detection}

Sequential detection [4] is an approach to reduce the average number of sensors required to reach a decision is also studied comprehensively during the past decades. In classical sequential detection, the basic idea is to minimize the sensing energy by minimizing the average sensing time, subject to constraints on the probability of false alarm and missed detection. Sequential detection has been adopted to reduce the sensing time in single radio spectrum sensing. However, multi 
sensor versions of sequential detection, i.e., cooperative or distributed sequential detection, are encountered more frequently in the field of spectrum sensing, since they provide the ability to significantly reduce the energy consumption of the overall system. Intrinsic to every sequential sensing scheme, is a stopping rule and a terminal decision rule. The stopping rule is a function that determines when to stop collecting observations and therefore is a random variable. The terminal decision rule dictates which decision has to be made after the sequential test has stopped.

\subsection{Confidence Voting}

Confidence voting [5] is a voting scheme based on users own confidence. Users vote only when they are confident about their spectrum sensingresults. The idea is that every user should refrain from sending unreliable information and cast its votes only when it has confidence.. If its spectrum sensing results accord with consensus, it gains its confidence, otherwise, it loses its confidence. When a users confidence level drops below a threshold, it considers itself as unreliable and stops sending its results. Once its confidence level passes beyond the threshold, it rejoins the voting. The main advantage of this scheme is that it saves energy by avoiding unnecessary transmission. In this scheme, the sent-out information can be either hard decision or soft decision.

\subsection{Cluster Collect Forwarding}

In Cluster Collect Forward scheme [5], The first step is to form clusters based on geometric locations, i.e., users who are close together form a cluster. After that, one of the member users is randomly chosen to become the cluster head. To avoid draining a users power quickly, cluster members take turns to become the cluster head. Cluster heads serve as local vote-collector (LVC). After users finalize its spectrum sensing, they transmit results to their local vote collectors. Vote-collectors add its own vote to the received votes and then forward the results (total number of votes or total scores) to the central vote collector (CVC). Thus, every local votecollector takes turns severing as CVC to prolong the overall lifetime. The CVC $\mathrm{X}$ is responsible for collecting and counting votes before announcing the total vote counts or weighted results back to local vote-collectors. LVCs then broadcast the final voting result to their cluster members.

\subsection{Energy Efficient Compressive Sensing}

Due to the emphasis on 'Green Wireless Communications' recently, energy efficiency is an aspect that must be dealt with in practical CR systems. This is especially so in wideband CR networks which operate in the over $\mathrm{GHz}$ regime and consequently conventional sampling and signal acquisition becomes energy costly from a hardware point of view. Hence Compressive sampling [6] has been proposed for spectrum sensing in CR networks recently. The basic concept is that if most of the data that is acquired can be thrown away without any perceptual loss, why not measure only that part that will not be thrown away? Spectrum sensing is a computationally energy consuming task. Hence, compressive sensing becomes highly relevant in the context of cognitive radios. Especially in the wideband regime, conventional Nyquist rate sampling becomes prohibitively costly. Compressive sampling techniques can be used to reduce the sensing costs of higher dimensional signals by exploiting sparsity in some domain. The number of samples needed to reconstruct the sparse signal is decided by actual sparsity order of the signal, which is often unknown and dynamically varying. Hence sampling rate is chosen according to an upper bound of sparsity order. An energy efficiency comparison between conventional cyclostationary feature detection and the scheme based on compressive sensing shows a reduction in required SNR for the latter which translates into reduced transmit power and hence energy saving.

\subsection{Reinforcement Learning Based Energy Efficient spectrum Sensing}

Reinforcement learning [7] is a trial-and-error machinelearning approach in which the decision maker, called the agent, observes the state of the environment and chooses actions that lead to rewards and new states. Actions leading to desired outcomes are given higher rewards, which reinforce these actions, thus making them more likely to be chosen again in similar situations in the future. Consequently, in reinforcement learning, the agent or agents are faced with the exploitation versus exploration tradeoff, i.e., whether to exploit the current best action or to explore other actions in hope of finding a better one. Energy efficiency is achieved by minimizing the number of assigned sensors per each sub band under a constraint on miss detection probability. The Reinforcement Learning Based Spectrum Sensing Policy balances between exploring and exploiting different parts of the radio spectrum and different sensing assignments.

\subsection{History Assisted energy efficient Spectrum sensing}

The history assisted energy efficient spectrum sensing scheme [8] employs an Iteratively developed history processing database. The usage of history helps predicting PU activity and results into reduced spectrum scanning by SUs thereby improving the sensing related energy consumption. Despite the fact that continuous scanning of the spectrum can fully capture the opportunities for the SUs, however it incurs costs in terms of increased energy consumption and sensing time. The history assisted spectrum sensing technique employs a database to process the spectrum sensing history and help SUs make a decision towards utilization ofan empty space or to perform continued spectrum sensing. It is shown that the increased history utilization helps SUs conserve energy during the spectrum sensing.

\subsection{Wireless sensor network assisted Cognitive Radio Networks}

An energy efficient network architecture that consists of ad hoc (mobile) cognitive radios(CRs) assisted by infrastructure wireless sensor nodes can reduce the energy consumption by the cognitive radios as the spectrum sensing is done solely by the sensor network. Here sensor nodes within communications range of each $\mathrm{CR}$ are grouped into a cluster and the clusters of CRs are regularly updated according to the random mobility of the CRs. . An ad hoc CR, which is a cluster head, is surrounded by a cluster of infrastructure sensor nodes within one-hop communication range of the CR, and each cluster is further partitioned into subsets. The energy consumption is reduced by dividing each cluster into disjoint subsets with overlapped sensing coverage of primary user (PU) activity. Sleep wake scheduling for the subsets based on the statistical behavior of the PU can impart further energy efficiency of the CRN [9].

\subsection{Energy efficiency through adaptive spectrum probing}

There is an optimal spectrum sensing interval which reduces total energy consumption. In the an adaptive spectrum sensing time interval strategy, SUs can adjust the next spectrum sensing time interval according to the current spectrum 
sensing results (namely, channel status).Its a dynamic spectrum sensing strategy in which the next spectrum sensing time is adaptive and based on current spectrum sensing results. That is, spectrum sensing time interval is not fixed, according to the current sensing result [10].

\subsection{Trust based energy efficient spectrum sensing}

The energy efficient collaborative spectrum sensing scheme based on trust management reduces the total number of sensing reports exchanged between the secondary users(SUs) and the secondary user base station (SUBS). The trust based Energy efficient spectrum sensing consists of two phases [11]:Phase I: Based on the trust values, the FC chooses a set of SUs which are to sense the band and transmit their reports to the FC in the mini time slots. The FC will broadcast a message containing the list of the chosen SUs. The FC fuses the reports from the chosen SUs with its own local decision and broadcasts the intermediate decision to all SUs.Phase II: If an SU disagrees with the intermediate decision, it can so indicate via a transmission in its designated mini time slot; otherwise, the SU remains quiet. SUs are synchronized to listen to the FCs broadcasts in Phase I.The information contained in the band-state matrix sent to SUBS can be used to evaluate a trust value for each SU, based on its accuracy in previous time slot.

\subsection{Time division energy efficient spectrum sensing}

Time-Division Energy Efficient (TDEE) sensing scheme is [12] in which the sensing period is divided into an optimal number of timeslots and each Secondary User (SU) is assigned to detect a different channel in one time-slot. An important advantage of TDEE is that the SUs do not need to exchange the control messages for the acknowledgement of a successful cooperation, leading to substantial energy. saving without compromising sensing accuracy. The time-division energy efficient sensing scheme allows a single SU to detect a number of different channels within one sensing period. The SU detects channels in time slots according to the channel information. The sensing-transmission operation of each SU is on a frame-by-frame basis in CR. The whole time frame can be divided into two parts: sensing and transmission. Each frame has a time duration $\mathrm{T}$, within which the SUs sense the channel for the duration of Ts. If the SUs do not detect signal from any PU over its channel, the remaining duration of the frame $\operatorname{Tr}=\mathrm{T}-\mathrm{Ts}$ is used for data transmission on an available channel.

\section{CONCLUSION}

In the past few years, with the growing demand for spectrum in various wireless applications and due to the inefficient spectrum utilization, there is a need to efficiently utilize the limited spectrum. Cognitive Radio plays an important role in such scenario and spectrum sensing is a vital aspect in CR. In this paper various energy efficient spectrum sensing methods are studied. The usage of these techniques are application dependent. One can select a suitable sensing technique according to their application but they are not without demerits. The Energy efficient spectrum sensing techniques increases the overall network life time of the cognitive radio networks. But they are with their own merits and demerits. In censoring, the number of transmissions is reduced and so is the transmission energy but the scheme is not without demerits. They are optimum selection of the two thresholds is difficult, FC in makes no decision when it receive no results from CR's and it Performs well with only Energy Detection.
Yet another technique called sleeping was discussed where the cognitive radios that are asleep do not waste any sensing or transmission power but there is a chance of an available Spectrum hole going missed. The main advantage of confidence voting was that it saves energy by avoiding unnecessary transmission but when many users have bad channels, voting can lead to wrong decision. In CCF scheme, energy saving in achieved not only by clustering but also by combining data before forwarding but more clusters needs more long range communication between LVCs and CVC and more clustering overhead, which are undesirable. History assisted technique has an advantage that reduced SS saves energy but increased delay and loss of quality for time sensitive traffic is a demerit. In WSN assisted CRNs main advantage is that no energy is wasted by CRN through SS except for the cost of a dedicated sensor network. Trust based SS has an advantage that only lesser number of sensing reports are required. In Time division Energy Efficient Spectrum Sensing scheme, energy is saved because no need to share control messages to all SUs, increase network throughput with guaranteed sensing accuracy. But if more cooperative SUs are involved, the exchanging with the fusion centre needs longer exchanging time. In RL based SS energy efficiency is achieved by minimizing the number of assigned sensors per each sub band under a constraint on miss detection probability but RL applications in clustering algorithms is still at infancy stage.

Recent trends of EE SS are utilizing history or employing a dedicated WSN for SS. Integration of different techniques together, like censoring combined with sleeping can further improve energy efficiency. Censoring, Sleeping, CF and CCF are primitive techniques and fails to work well with more complex techniques like feature detection. In delay sensitive applications History assisted SS and TDEESS cannot be used as they cause additional delay, while WSN for CRN works well with minimum delay. Throughput is maximum with TTEESS scheme when compared to other EESS schemes. Residual energy per node is taken care the most in History assisted SS while other techniques concentrates only on overall system life time. RL is a better way of EESS compared to others but is still at the infancy stage. Apart from the cost of a dedicated WSNs, WSN sensing the spectrum for CRNs is the best of all techniques as energy wasted by CRN in SS is almost zero.

\section{ACKNOWLEDGMENT}

This work is supported by Center for Engineering Research and Development (CERD), Govt. of Kerala, India under the project Research Seed Money (RSM) No. C1/RSM 99/2014.

\section{REFERENCES}

[1] radios more personal", IEEE Personal Commun. Mag., vol. 6, no. 4,pp. 13-18, Aug. 1999.

[2] Nabil Giweli, SeyedShahrestani Mitola, J. and J. Maguire, G. Q., "Cognitive radio: making software and Hon Cheung; "Spectrum sensing in cognitiveradio networks :Qosconsiderations", CS IT-CSCP 2015 ,pp. 0919

[3] SinaMaleki, "Energy-Efficient Spectrum Sensing for Cognitive Radio Networks”, 2015 ,CSIT CSCP,pp 09-19

[4] Erik Axell, Geert Leus, Erik G. Larsson, and H. Vincent Poor, "Spectrum Sensing forCognitive Radio"; IEEE Signal Processing Magazine,May2012. 
[5] Chia-hanLee,Wayne Wolf, "Energy Efficient Techniques for Cooperative SpectrumSensing in Cognitive Radios", IEEE CCNC 2008 proceedings, pp 128-134.

[6] ViswanathanRamachandran, AlicCheeran, "Improvement of Energy Efficiency ofSpectrum Sensing Algorithms for Cognitive Radio Networks using Compressive SensingTechnique",International Conference on Computer Communication and Informatics (ICCCI -2014), Jan, 2014,pp 1-6.

[7] Jan Oksanen, JarmoLunde, VisaKoivunen, "Reinforcement learningbasedsensing policyoptimization for energy efficient cognitive radio networks", 2011 ElsevierJ. Oksanenetal./Neurocomputing80(2012), pp102 - 110

[8] Tazeen S. Syed, Ghazanfar A. Safdar, "History assisted Energy Efficient Spectrum Sensing for Infrastructure based Cognitive Radio Networks", IEEE Transactions on vehicular technology, January 2016,pp 1-15.
[9] Muhammad Usman, Member, IEEE, DangsooHar, Senior Member, IEEE, and InsooKoo, Member, "IEEE,Energy-Efficient Infrastructure Sensor Network for Ad HocCognitive Radio Network", IEEE Sensors Journal, January 2016, 1-13

[10] Zesheng Chen, Chao Chen., "Adaptive energy efficient spectrum probing in cognitiveradio networks" Elsevier Z. Chen, C. ChenAd Hoc Networks (2014) ,pp.256-270

[11] S. Ali Mousavifar and Cyril Leung, "Trust-Based Energy Efficient Spectrum Sensingin Cognitive Radio Networks",IEEE journal2013, pp 1-6.

[12] Yi Liu, Rong Yu, Shengli Xie,Yan Zhang, and Victor C.M. Leung, "Energy-Efficient Spectrum Discovery for Cognitive Radio Green Networks", 18th International conference on Telecommunication, ,pp 64 to 74, IEEE 2011. 